\title{
Sessile Drop in Microgravity: Creation, Contact Angle and Interface
}

\author{
David Brutin • ZhiQuiang Zhu • Ouamar Rahli • \\ JingChang Xie • QuiSheng Liu • Lounes Tadrist
}

Received: 24 February 2009 / Accepted: 26 May 2009 / Published online: 6 June 2009

(C) Springer Science + Business Media B.V. 2009

\begin{abstract}
We present in this paper the results obtained from a parabolic flight campaign regarding the contact angle and the drop interface behavior of sessile drops created under terrestrial gravity $(1 \mathrm{~g})$ or in microgravity $(\mu \mathrm{g})$. This is a preliminary study before further investigations on sessile drops evaporation under microgravity. In this study, drops are created by the mean of a syringe pump by injection through the substrate. The created drops are recorded using a video camera to extract the drops contact angles. Three fluids have been used in this study: de-ionized water, HFE-7100 and FC72 and two heating surfaces: aluminum and PTFE. The results obtained evidence the feasibility of sessile drop creation in microgravity even for low surface tension liquids (below $15 \mathrm{mN} \mathrm{m}^{-1}$ ) such as FC-72 and HFE7100. We also evidence the contact angle behavior depending of the drop diameter and the gravity level. A second objective of this study is to analyze the drop interface shape in microgravity. The goal of the these experiments is to obtain reference data on the sessile drop behavior in microgravity for future experiments to be performed in an French-Chinese scientific instrument (IMPACHT).
\end{abstract}

\footnotetext{
D. Brutin $(\varangle) \cdot$ O. Rahli $\cdot$ L. Tadrist

Laboratoire I.U.S.T.I., Ecole Polytechnique Universitaire de Marseille, Technopôle de Château Gombert, 5 rue Enrico Fermi, 13453 Marseille, France

e-mail: david.brutin@polytech.univ-mrs.fr
}

Z.Q. Zhu · J.C. Xie · Q.S. Liu

National Microgravity Laboratory of China, Institut of Mechanics, Chinese Academy of Sciences,

Bei Si Huan Xi Road, 15, Beijing 100190, China
Keywords Wettability - Contact angle • Sessile drop • Drop interface $\cdot$ Drop creation

\section{Introduction}

Evaporation is a process commonly met in nature with salt-water, spots of coffee, weed killers and industrial processes like cooling, welding, drying of paintings, desalinization, micro-electronics and evaporators. The heat transfer during evaporation is much larger than that obtained with traditional techniques without phase change. During evaporation the mass transfer and the heat transfer along the fluid interfaces and their optimization are often a vital objective. The control of these processes where the liquid-vapor phase change intervenes is still badly known at the present time even if it is largely used in industry to transfer important heat fluxes. A clear identification of the physical phenomena and a better knowledge of the role of the parameters should make it possible to work out apparatuses at the same time more powerful and with sizes more reduced than those existing at the present time. The modeling of the mass and heat fluxes from the liquid to the interface gas poses many problems related to the nature of the liquids and gases in contact, and the configuration and geometry of the interface. It is also necessary to or not take account of the presence or not of surfaceactive, volatile liquids and of the incondensable ones. We showed within the framework of the thesis of Grandas (2004) that the evaporation of a drop posed on a support is strongly localized at the level of the triple line. At the liquid-gas interface level, this mechanism is controlled being given the various movements induced by the effects of gravity and surface tension. In 


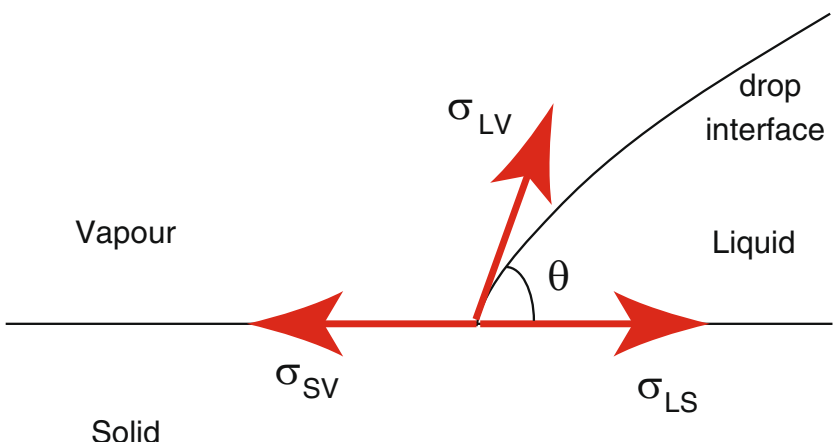

Fig. 1 Contact angle definition for a sessile drop

particular the influence of the radius of curvature is currently badly known. Evaporation is for example used to create ordered mono layers nanoparticles deposition for industrial applications (Bigioni et al. 2006). The convection inside the drop enable an accumulation at the drop interface of particles initially dispersed inside the fluid. The drop pinning process on the surface is possible through the particles adhesion on the surface. The evaporation phenomena can generate temperature variations in the vicinity of the solid surface and along the drop interface. The Marangoni effect can produce instabilities at the interface. The purpose of the experiments presented here is to analyze the mechanisms of drop creation and its consequences in the solid, liquid and the gas phases as the variations of the contact angle and dimensions of the drop (profile and perimeter of contact). An important area to take into account in the analysis is the contact line. This line represents the location where the three interfaces meet. In this zone, an intense evaporation takes place.

The drop evaporation research subject is strongly related to the vicinity of the triple line. Thus, the contact angle of a sessile drop on a heated surface is of great important to predict and analyze the drop evaporation (Fig. 1). In the frame of an instrument developed for satellite experiment on drop evaporation in microgravity; in a first time, we investigated here the contact angle behavior in normal, hypergravity and microgravity of sessile drop on different wall nature. The prediction of contact angle in microgravity is not investigated in the community based on the literature review of Table 1. All the studies found are performed under normal gravity. Ponter and Boyes (1972) firstly studied the sessile drop contact angle behavior depending of the drop diameter for a pressure range of 50 to 760 Torr. They worked with saturated vapor conditions only at the boiling point on copper surfaces. They did not observed any change of the contact angle for drop bigger than $5 \mathrm{~mm}$. The changes were observed for small drop below $5 \mathrm{~mm}$ when the competition between gravity and surface tension exist.

Bernardin et al. (1997), Panwar et al. (2003), Grandas et al. (2005) and Takata et al. (2005) investigated water sessile drops behaviors on different surfaces such as glass, polycarbonate, PTFE, $\mathrm{TiO} 2$ or $\mathrm{SiO} 2$. All authors observe contact angle behaviour depending on the thermal properties and surface roughness of the substrate. Gajewski (2008) evidence the hysteresis effect on the sessile drops contact angles with increasing drop diameters and decreasing diameters even on metallic surfaces. They evidence that aluminum and stainless steel loose their hydrophobic properties during the drop diameter increase or decrease procedure while they do not observe this behaviour with copper and brass. They confirm that the critical contact angle value depends on the chemical properties of the surface and on the surface roughness. Whyman and Bormashenko (2009) present in their paper, a model

Table 1 Publications related to sessile drop contact angles [several approaches using numerical simulation (num.), experiments (exp.) or theoretical investigations $(t h)$.

\begin{tabular}{llll}
\hline Author (reference) & Approach & Liquid used & Solid surface \\
\hline Ponter and Boyes (1972) & Exp. & Water & Copper \\
Bourges-Monnier and Shanahan (1995) & Num., Exp. & Water, $n$-decane & PTFE, glass, epoxy \\
Chandra et al. (1996) & Exp., Th. & Water with surfactant & Stainless steel \\
Bernardin et al. (1997) & Exp. & Water & Aluminium \\
Zhang and Chao (2001) & Exp. & Silicon oil, Freon 113, Ethanol & Aluminized glass plate \\
Panwar et al. (2003) & Exp. & Water & Glass, polycarbonate \\
Grandas et al. (2005) & Exp. & Water & PTFE \\
Takata et al. (2005) & Exp. & Water & TiO $_{2}, \mathrm{SiO}_{2}$ \\
Frassy et al. (2006) & Exp., Num. & Water & $\mathrm{SiO}_{2}$, silane, polycarbonate \\
Letellier et al. (2007) & Th., Exp. & Water, silicon oil & Teflon, FC725, PMMA, silica $^{\text {Gajer }}$ \\
Gajewski (2008) & Exp. & Water & Aluminium, brass, stainless steel \\
Whyman and Bormashenko (2009) & Th., Exp. & Water & Polyethylene \\
Kulinich and Farzaneh (2009) & Exp. & Water & Super-hydrophobic polymer \\
\hline
\end{tabular}


for the calculation of the shape and contact angles of heavy droplets, the model is relevant when heavy droplets are investigated. Consequently, this model is relevant when gravity plays an important role on the drop shape. The authors provide comparison with experimental results for very small drops and conclude that for these light drops the spherical model is still relevant however when the drop diameter reach the capillary length, the spherical model is no more accurate to describe the drop shape interface. Kulinich and Farzaneh (2009) evidence different evaporation modes on super-hydrophobic polymer surfaces (with drops contact angles higher than $150^{\circ}$ ). They also show different hysteresis of contact angle. They observe that water droplets evaporate faster on surfaces with high contact angles hysteresis.

These papers evidence that the drop contact angle depends on several parameters such as the chemical and physical substrate properties. The geometrical surface properties can also deeply modify the contact angle of a sessile drop. Furthermore, these studies evidence that a hysteresis problem exist when the drop contact angle is measured for increasing drop diameter and decreasing.

\section{Experimental Set-Up}

The experimental setup is designed to enable a sessile drop creation under microgravity. A test cell is located inside a confinement box to create a drop with a thermally controlled environment.

\section{The Rack and the Test Cell}

The rack is composed of a confinement box thermally controlled in which a test cell is used to evaporate sessile drops. A $700-\mu \mathrm{m}$ diameter hole is performed thought the heating surface inside the test cell to create the drop by injection at low mass flow rate, as shown with Fig. 2. The injection mass flow rate is a parameter to be determine experimentally in microgravity, to avoid jet formation at high mass flow rate. If the mass flow rate is too low, the drop creation time will be to
Fig. 2 Confinement box and its equipment to achieve drop creation on aluminum or PTFE

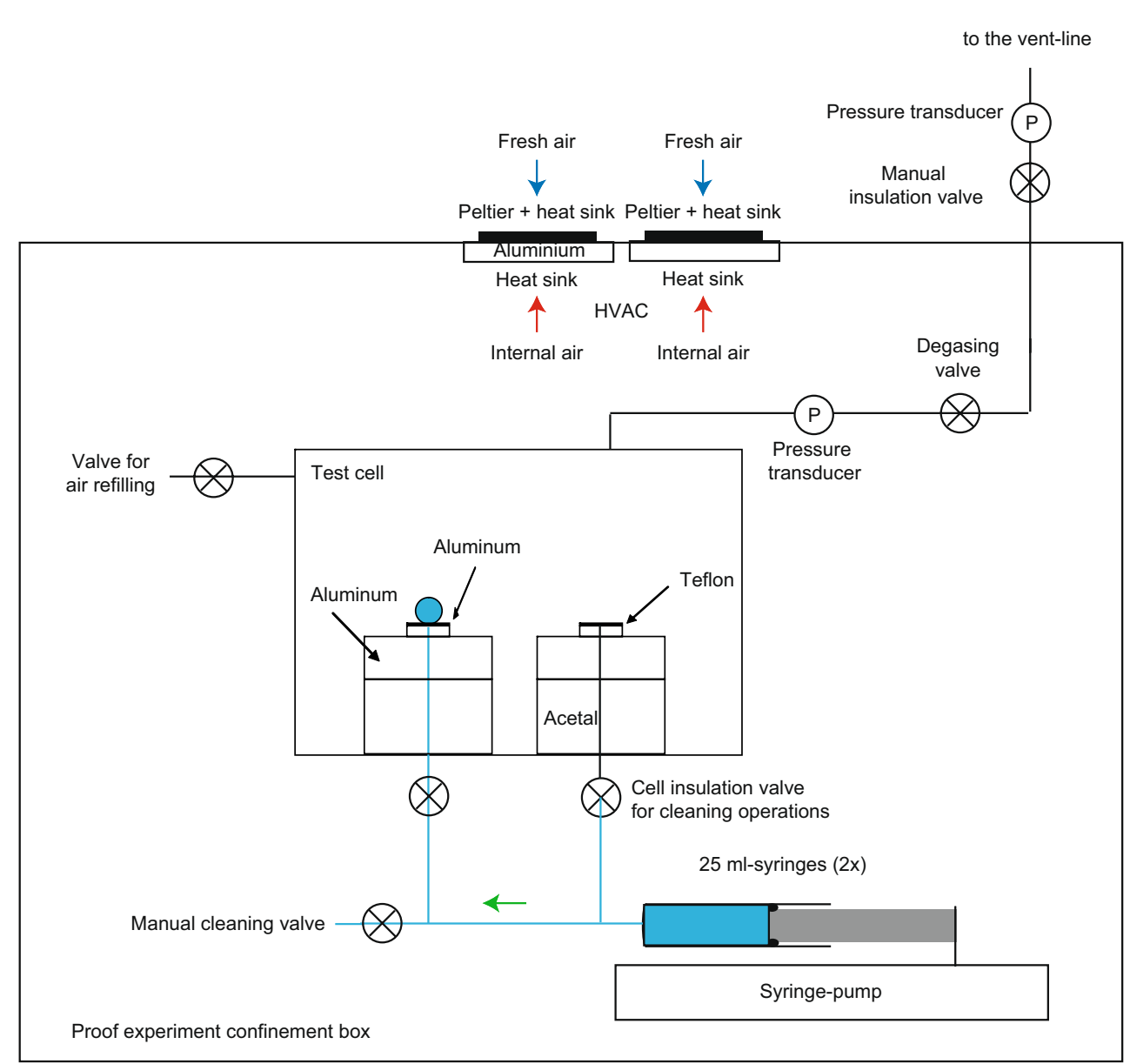


important compared to the microgravity time (20 s). The substrate temperature can be modified using heating cartridges. The surface temperature can be increased in temperature of $+10^{\circ} \mathrm{C},+15^{\circ} \mathrm{C},+20^{\circ} \mathrm{C},+30^{\circ} \mathrm{C}$ from the cell air temperature which is for all experiments at $20^{\circ} \mathrm{C}$.

The drops are created in terrestrial gravity or microgravity to measure and observe the contact angles behaviour. This is possible since a $10 \mathrm{~s}$ period at $1.8 \mathrm{~g}$ followed by 10 more seconds at $1.5 \mathrm{~g}$ are obtained before microgravity. Furthermore, the experiments are performed to confirm the sessile drop creation feasibility in microgravity even for low surface tension fluids (FC-72 and HFE-7100). Only one fluid is used for each flight day to ensure to keep clean pipes. Under terrestrial gravity, the drop during the creation is maintained on the surface by gravity. Under microgravity, the drop will be maintained on the surface mainly due to the surface tension and is slightly influenced by the microgravity. For low surface tension fluids, the drop could detach from the surface if the vibration level is too important or if the surface tension is too low.

\section{Data Acquisition}

The experimental rack is equipped of a visible camera $(800 \times 600$ pixels) to record the drop creation. The test cell is regulated in the temperature and pressure which are recorded using respectively K-type thermocouples and a differential pressure sensor. The heating substrate temperature is regulated in temperature using a PT-100 sensors associated with a PID regulator at $\pm 0.1^{\circ} \mathrm{C}$. The substrate heating is obtained using two heating cartridge of $15 \mathrm{~W}$ each.

\section{Working Fluids}

The fluid properties used in this paper are presented in Table 2. Using the fluid physical properties, it is possible to determine the capillary length for the 3 gravity levels studied. The capillary length is useful to determine the critical diameter between the gravitydeformable drop and non-deformable drop. The critical diameter is about two times the capillary length give in Table 2. When the drop is very small (droplet) the surface tension is mainly dominating the drop interface curvature. The gravity for this situation have almost no effect on the drop interface. However, when the drop diameter is bigger than the capillary length, the drop interface is strongly influenced by gravity effects. Water is used for it's high surface tension value but have a high saturation temperature and phase change enthalpy. On the other way, FC-72 and HFE-7100 are tested for their low surface tension. Theses two fluids have also a low saturation temperature and phase change enthalpy which will be useful for the next step which concern the mass flow rate of evaporation.

\section{Wettability}

To easily access the contact angle by image processing, we need to obtain a picture with a diameter/height ratio of about 1 . If the drop diameter is to important in front of the drop height, the contact angle will be difficult to determine due to the difficult determination of the drop interface. Consequently, if the liquid/surface wettability is low, which correspond to wetting situation observed for FC-72 and HFE-7100, the liquid will spread on the surface, so that the contact angle will be below $10-15^{\circ}$. However for non-wetting situations (water and PTFE or aluminum) the drop ratio (drop height/drop radius) is close to 0.5 , so that the contact angle is greater around $90^{\circ}$. These two situations are summarized in Fig. 3.

\section{Image Processing}

A software program was developed to determine some physical parameters of the experimental liquid drops from the shapes of the drops, such as the volume, surface area and contact angle. And the program improved the classical algorithm proposed by Rotenberg et al. (1983). From the acquired grey pictures, general contour points of a drop were obtained by edge detection. Then, a Laplace equation was constructed based on the contour of the drop. And the contour approach process was performed to approach the true contour

Table 2 Liquid physical properties and capillary lengths at $25^{\circ} \mathrm{C}$ and $1 \mathrm{~atm}$

\begin{tabular}{|c|c|c|c|c|c|c|c|c|c|c|}
\hline & $\varrho_{L}$ & $\varrho_{V}$ & $\mathrm{Cp}$ & Lv & $\lambda$ & $\mu$ & $\sigma$ & $L_{\mathrm{c}}(\mu \mathrm{g})$ & $L_{\mathrm{c}}(1 \mathrm{~g})$ & $L_{\mathrm{c}}(1.8 \mathrm{~g})$ \\
\hline & $\mathrm{kg} \mathrm{m}^{-3}$ & $\mathrm{~kg} \mathrm{~m}^{-3}$ & $\mathrm{~J} \mathrm{~kg}^{-1} \mathrm{~K}^{-1}$ & $\mathrm{~kJ} \mathrm{~kg}^{-1}$ & $\mathrm{~W} \mathrm{\textrm {m } ^ { 2 }} \mathrm{K}^{-1}$ & $\mathrm{~Pa} \mathrm{~s}$ & $\mathrm{mN} \mathrm{m}^{-1}$ & $\mathrm{~mm}$ & $\mathrm{~mm}$ & $\mathrm{~mm}$ \\
\hline Water & 997 & 0.59 & 4,180 & 2,449 & 0.606 & $0.89110^{-3}$ & 72.7 & 12.2 & 2.7 & 2.0 \\
\hline HFE-7100 & 1,520 & 2.84 & 1,183 & 111 & 0.068 & $0.56210^{-3}$ & 13.6 & 4.3 & 0.96 & 0.71 \\
\hline FC-72 & 1,680 & 4.35 & 1,100 & 88 & 0.057 & $0.63810^{-3}$ & 12.0 & 3.8 & 0.82 & 0.64 \\
\hline
\end{tabular}




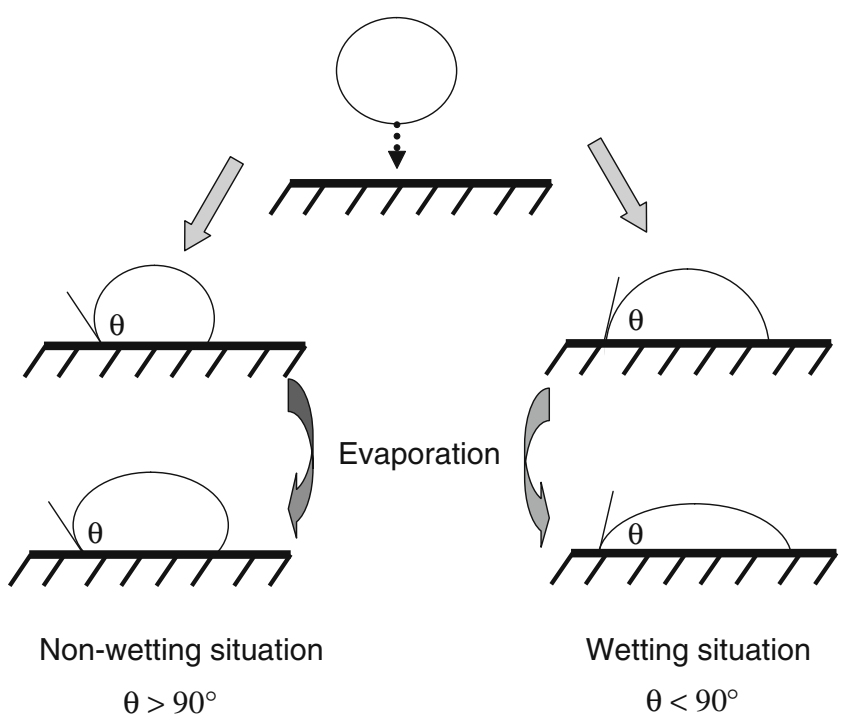

Fig. 3 Possible wetting situations when a liquid drop is posed on solid surface

by use of Newton-Raphson method and Rouge-Kutta method. The volume, surface area and contact angle of the drop is achieved according to the final calculated curve. More details could be found in Ning (2008). Figure 4 presents the geometry analyzed graph.

The equations used in the algorithm are:

$$
\begin{aligned}
& \frac{\mathrm{d} x}{\mathrm{~d} s}=\cos (\phi) \\
& \frac{\mathrm{d} z}{\mathrm{~d} s}=\sin (\phi) \\
& \frac{\mathrm{d} \phi}{\mathrm{d} s}=2+\frac{B o \times z}{4}-\frac{\sin \phi}{x}
\end{aligned}
$$

where $B o$ is the Bond number calculated based on $R_{0}$ which is the radius of curvature for the origin of the coordinates.

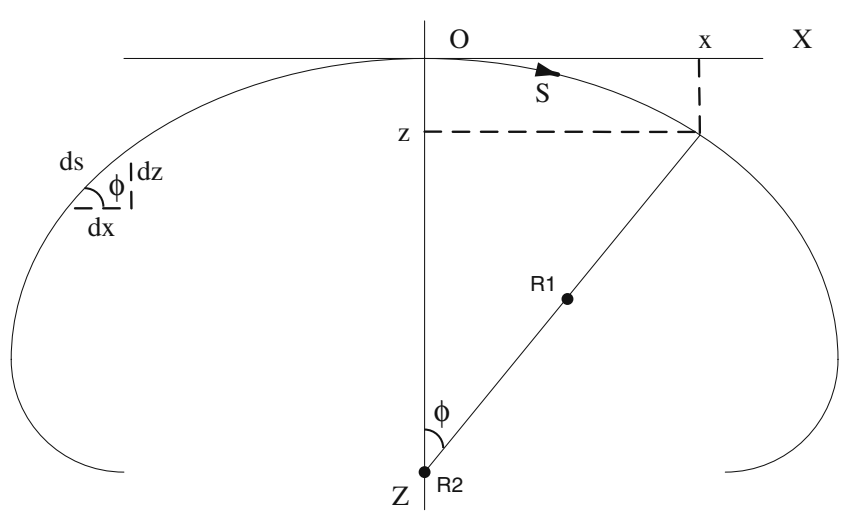

Fig. 4 Geometry analyzed graph
The quality of the picture influences evidently the precision of the calculated curve. The contour approach is performed under the hypothesis that the liquid drop is symmetrical. We only calculate the right half of the drop to minimize the integration time. Therefore, the liquid drop should be created to be as symmetrical as possible. Meanwhile, to minimize the fitting error, the substrate of the drop should be set to be horizontal and the axis of the CCD lens should be set along the center line of drop to distinguish the bottom line of the drop clearly.

\section{Data Analysis}

This section is mainly divided into the drop creation results and the created drop analysis (contact angle and interface equation). For each experiment, a typical set of data are recorded: a movie of the drop creation at $7.5 \mathrm{~Hz}$ which enable us to check the drop equilibrium for the contact angle measurement, the test cell air temperature and pressure, the acceleration of the three plane axis, the experiment conditions: substrate temperature, fluid and drop creation period. For each experiment, a data treatment is performed to obtain: the left and right sessile drop contact angle to check the drop symmetry, the drop volume, the drop wetting diameter.

Hereafter, we present the results obtained on water drops contact angle and drop creation with the 3 fluids. The contact angle measurement is not enough accurate for HFE-7100 and FC-72 based on the small contact angle observed (about $10^{\circ}$ ). Also, the best quality images have been obtained for water which is non-wettable on PTFE. The drop aspect ratio which is about 0.5 enable us to performed good quality pictures for the contact angle determination.

\section{Drop Creation in Microgravity}

To study sessile drop evaporation in microgravity, it is necessary to create these drops in microgravity. During parabolic flights, it is possible to use the $1 \mathrm{~g}$ period before the parabola to create the drop then to analyze the drop behavior (contact angle, wetting diameter and drop interface). However, in the frame of experiments in a scientific satellite, the drop need to be created without gravity. Depending on the surface tension, injection hole diameter and mass flow rate of injection, the liquid can create a jet. Due to the absence of gravity, only the surface tension can hold the drop on the surface.

On Fig. 5, we present a FC-72 drop creation in microgravity with an injection mass flow rate of $6.7 \mu \mathrm{L} / \mathrm{s}$ 
Fig. 5 FC-72 drop creation on PTFE in microgravity at $6.7 \mu \mathrm{L} / \mathrm{s}$
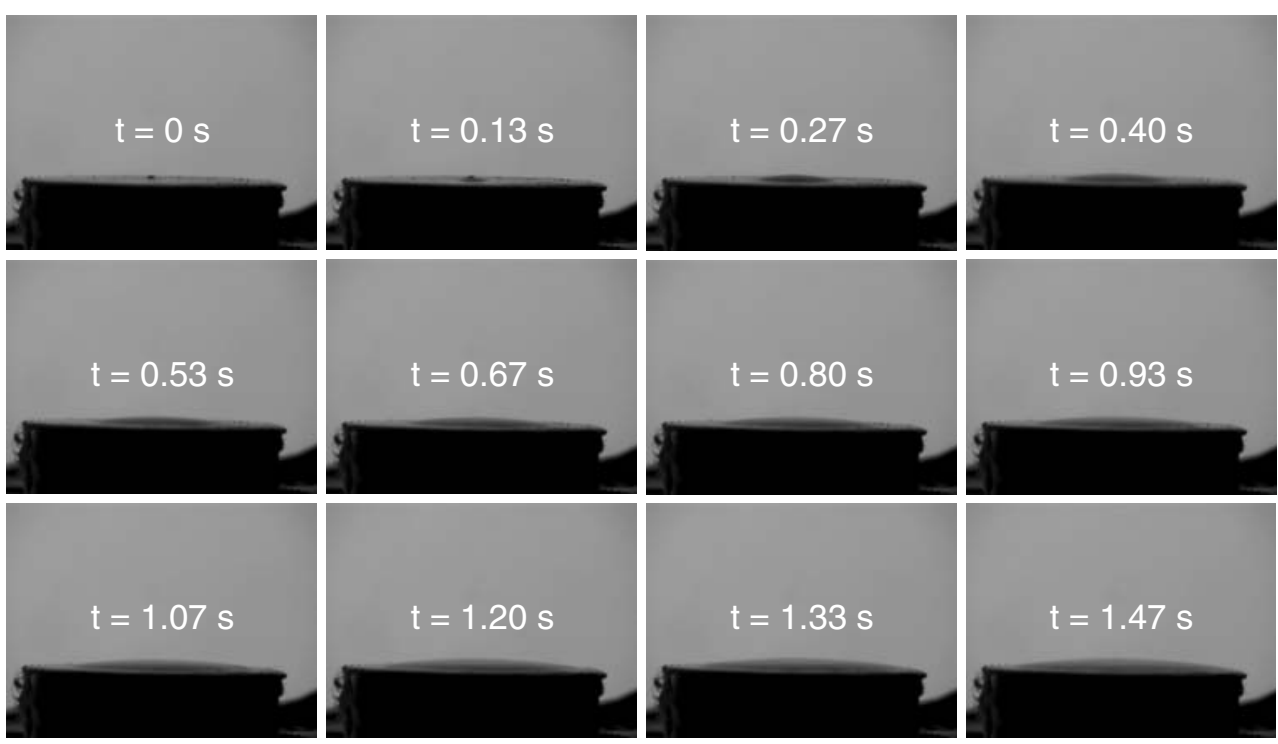

which correspond to an injection velocity at the injection hole exit of $0.017 \mathrm{~m} / \mathrm{s}$. The injection mass flow rate is low enough to avoid jet formation and is high enough in front of the evaporation mass flow rate. The small contact angle observed (below $10^{\circ}$ ) do not enable an accurate determination. The FC-72 drop wet the PTFE surface and the drop remained posed on the surface during the diameter increase until the liquid reach the edge of the substrate. The liquid clearly remain on the substrate during the drop creation which is less than $1.5 \mathrm{~s}$.

However, if the injection mass flow rate is too important (see Fig. 6), the interface is deformed during the drop formation. The injection mass flow rate is here 10 times greater (compared with Fig. 5) with $67 \mu \mathrm{L} / \mathrm{s}$. The injection jet which exit at $0.17 \mathrm{~m} / \mathrm{s}$ from the hole is visible since the first second of the drop creation. Meanwhile, the injection jet do not break the drop interface. As soon as the liquid reach the substrate boundary, the repealing effect enable the drop volume increase thought the increase of the contact angle. This contact angle, when the drop reach the boundary, do not reflect a static sessile drop contact angle as defined with Fig. 1. When the injection mass flow rate stops, the drop interface come back almost hemispherical.

\section{Drop Contact Angles}

The typical drop contact angle observed for 3 different levels of gravity are presented in Fig. 7. A water drop is created under terrestrial gravity then the drop is put under hypergravity with $1.8 \mathrm{~g}$ then $1.5 \mathrm{~g}$. Finally the drop is in microgravity during $20 \mathrm{~s}$. This sequence of gravity level changes which is presented in Fig. 7 evidence a particular behavior of the drop contact angle. Under normal gravity the drop contact angle is $95.3^{\circ}$, under hypergravity the contact angle reduce to $76.6^{\circ}$ the drop interface is also modified. However under microgravity, the drop contact angle increase to reach $80.8^{\circ}$ but remain below the $1 \mathrm{~g}$ value. The initial drop was created under terrestrial gravity, the triple line advanced over the substrate while gravity was acting for this situation. The consequence on the contact angle value should be different is the drop is created in microgravity when triple line behavior on the substrate is not influenced by gravity (Fig. 8).

To check the contact angle behavior under normal gravity on the surface used in microgravity, we performed reference experiments with water on PFTE during the flight before the parabola. Even with the plane vibration, we obtained the results present in Fig. 9 which evidence increasing contact angle with increasing drop diameters. The uncertainty on the drop diameter and contact angle measurement is good enough to observe the effect of the plane vibration on the points location. An increasing behavior of drop contact angle is observed with increasing drop diameter. This is in accordance with the literature results Yekta-Fard and Ponter (1989). The contact angle behavior with gravity evidence a classical behavior with for big drops (diameter greater than the capillary length). The range of contact angle varies from $70^{\circ}$ to $100^{\circ}$. When the contact angle measurement is realized on drop in microgravity (but created under normal gravity) the contact angles are more dispersed in the range $80^{\circ}$ to $91^{\circ}$ for the same range of drop diameter between 4 and $10 \mathrm{~mm}$ as shown 
Fig. 6 HFE-7100 drop creation on aluminum in microgravity at $67 \mu \mathrm{L} / \mathrm{s}$ : the interface is deformed due to the important injection mass flow rate

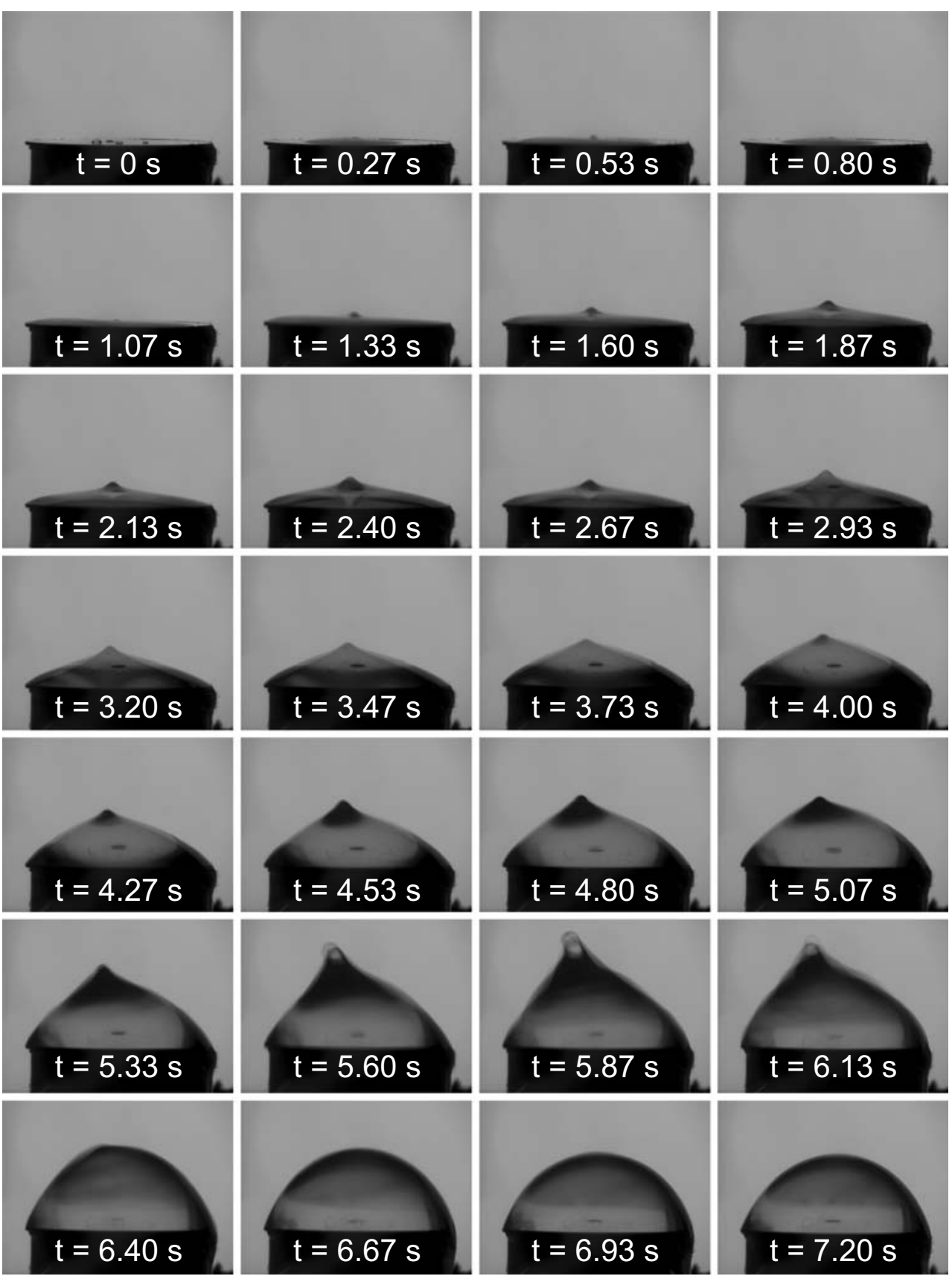

with Fig. 10. The contact angle in microgravity is very sensitive to the G-Jitter. We observe drop lateral oscillation due to G-Jitter which introduce contact angle variation of about $10^{\circ}$.

On Fig. 8, we present two drops of about the same base diameter but one is created under terrestrial gravity while the other one is created in microgravity. The left picture in Fig. 8 show a drop created during terrestrial gravity, then the drop is put under hypergravity then microgravity. The drop base diameter remain constant at $6.9 \mathrm{~mm}$, the contact angle in microgravity is $93^{\circ} \pm 3^{\circ}$. On the same substrate (PTFE) with the same fluid (water) and the under the same experimental conditions (cell temperature and pressure), a drop is created during microgravity. This drop obtained in microgravity have a base diameter of $6.5 \mathrm{~mm}$ and a contact angle of $107^{\circ} \pm 1^{\circ}$. Both drops interfaces are different due to the contact angle value which induce a totally different drop volume: $83 \mu \mathrm{L}$ for the drop created under normal gravity and $116 \mu \mathrm{L}$ for the drop 


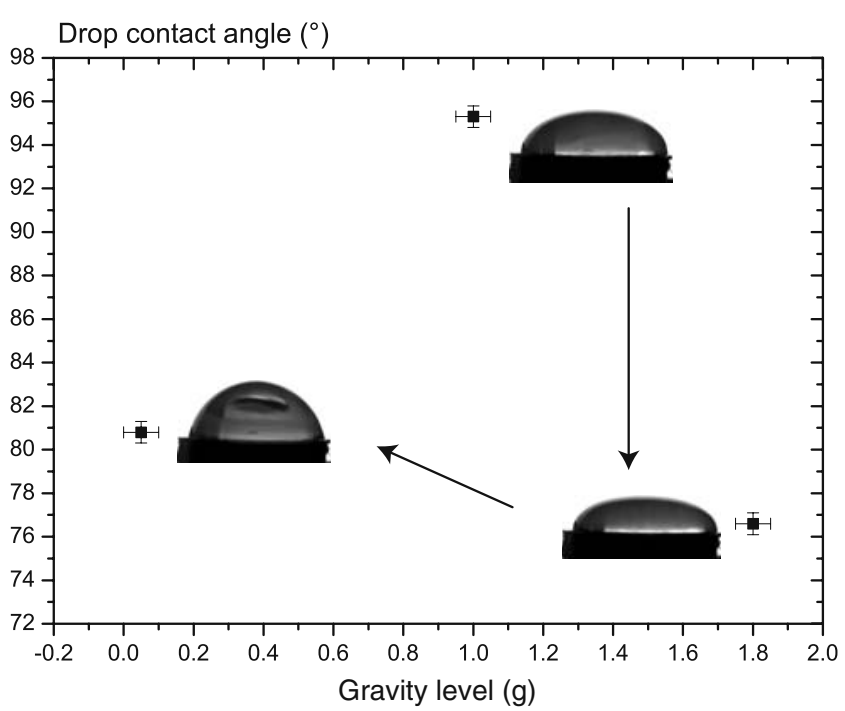

Fig. 7 Water drop contact angle on PTFE during a parabola

created during the microgravity. When the drop is created under normal gravity, the triple line advance over the surface and the fluid wet the surface based on the two physical forces: gravity and surface tension. The fluid is "pushed" on the substrate by the gravity which gives a contact angle of $93^{\circ}$. However, when the drop is created in microgravity, the triple line advance on the surface without the influence of gravity, the fluid is not "pushed" into contact on the surface. Consequently, the liquid seems not to wet the surface in the same way compared to a situation with gravity. The surface properties such as roughness, physico-chemical interactions and gas trapping between the substrate and the liquid seems to play a important role on the contact angle in microgravity. These different behaviors and contact angles with or without gravity when a drop is studied are of great importance to predict the drop volume for future microgravity studies in a satellite or on-board the ISS. The drop volume prediction is requested for the tank design for example; while the contact angle prediction is requested both for an experiment design and for numerical studies.
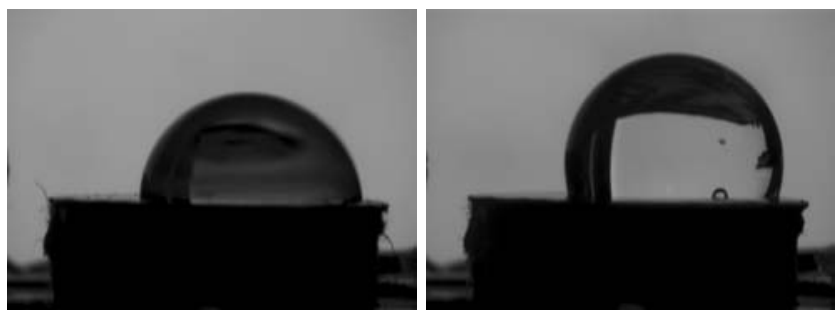

Fig. 8 Water drops on PTFE: drop created during $1 \mathrm{~g}$ on left picture, drop created during microgravity on the right picture

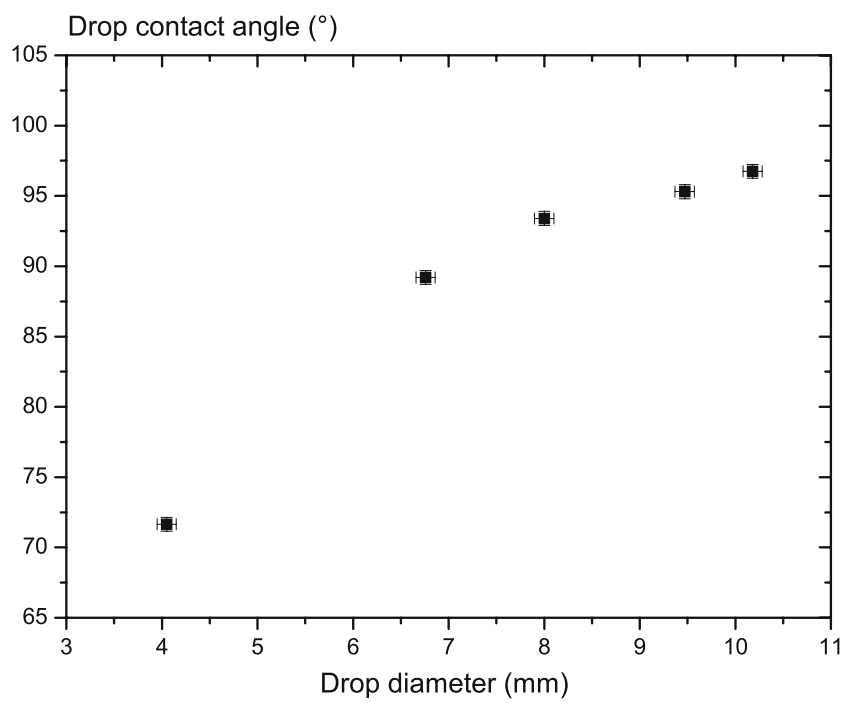

Fig. 9 Water drop contact angle on PTFE under $1 \mathrm{~g}$ for several drop diameters

\section{Sessile Drop Interfaces}

The drop interface equation is given by a pressure balance (Eq. 4). At the hydrostatic equilibrium, the overpressure inside the drop is given by the balance of the Laplace pressure and the hydrostatic pressure.

$P_{0}-\varrho g z=\sigma\left(\frac{\mathrm{d}^{2} z}{\mathrm{~d} x^{2}}+\frac{1}{x} \frac{\mathrm{d} z}{\mathrm{~d} x}\right)$

where $L_{\mathrm{c}}=\sqrt{\frac{\sigma}{\varrho g}}$ is the capillary length and $P_{0}$ the pressure at the top of the drop. The equation to obtain the interface profile is solved in polar coordinate as defined in Fig. 4 (Aussillous 2002). Two parameters are

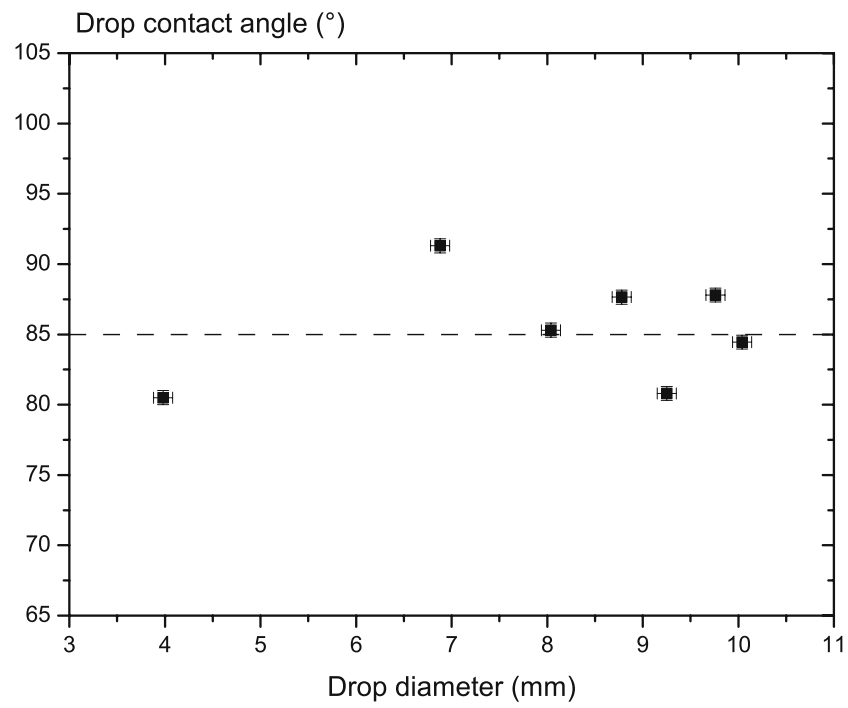

Fig. 10 Water drop contact angle on PTFE under microgravity for several drop diameters 


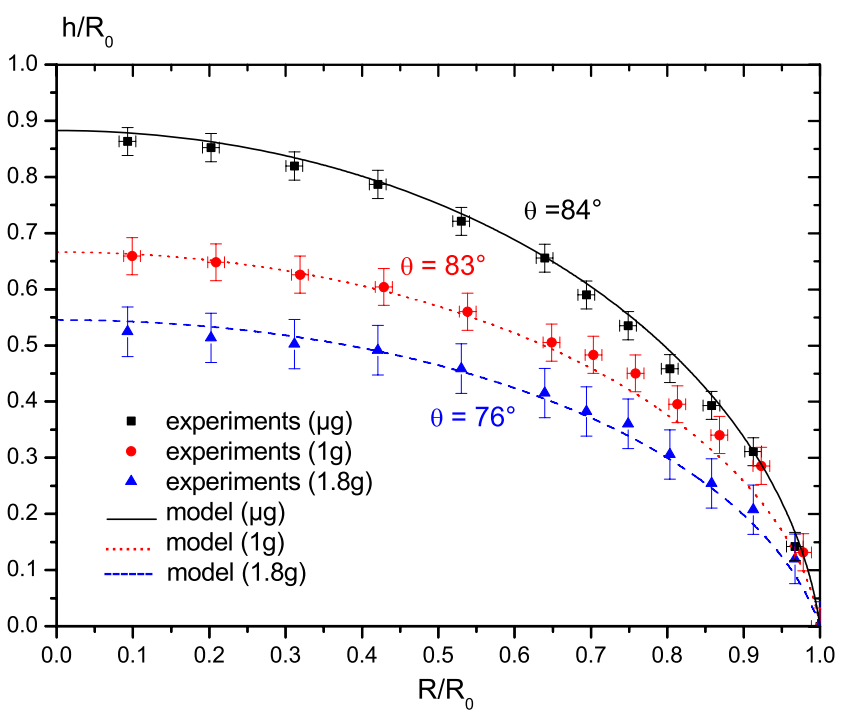

Fig. 11 Water drop non-dimensional interface equation for three level of gravity compared to the Laplace-hydrostatic model $\left(\mathrm{R}_{0}=4 \mathrm{~mm}\right)$

used to solve numerically Eq. 4: the pressure at the top of the drop $\left(P_{0}\right)$ and the drop contact angle $(\theta)$. These two parameters have to be modified to verify the two other physical parameters which are the drop base diameter and the drop height. Consequently, a drop contact angle is obtained.

Based on the images obtained for three gravity levels during the parabolic flight, we extract the experimental drop interfaces and plot them on Fig. 11. The contact angles obtained for these 8-mm diameter drops are: $85.3^{\circ}$ in microgravity, $93.4^{\circ}$ under normal gravity and $89.5^{\circ}$ under hypergravity. On the same figure, we plot the drop interface modeled using the LaplaceHydrostatic equation. We succeed to predict all three drop interfaces within the error margin and we obtain for the contact angle: $84.0^{\circ}$ in microgravity, $83.0^{\circ}$ under normal gravity and $76.0^{\circ}$ under hypergravity. This increasing variation of the contact angle with decreasing gravity level is in accordance with the LaplaceHydrostatic equilibrium. We obtain a good agreement for the contact angle only in microgravity. When gravity exist, a gap of more than $10^{\circ}$ exist. This can be attribute to the difficult repeatability of contact angle experiments with gravity fluctuation and plane vibrations.

\section{Conclusions and On Going Work}

In the frame of a long term research plan on sessile drop evaporation in microgravity; we performed basic experiments on sessile drop creation in microgravity. We focused on the drops contact angle and drop interface depending of the gravity levels. We observe that under terrestrial gravity and hypergravity, the contact angle increase to reach a constant value from small droplets to big drops; we observed in microgravity a different behavior with almost a constant contact angle very sensitive to the gravity level even for small drops with a diameter smaller than the capillary length. On the drop creation, we confirmed the feasibility to create posed drop under microgravity conditions even with low surface tension fluids such as FC-72 and HFE-7100. We evidence two different contacts angles considering water drop creation : if the drop is created under normal gravity then is put under microgravity the contact angle can be $10^{\circ}$ lower compared to the same drop created in microgravity.

The next step is now to investigate the drop contact angle with a better gravity level which can be obtain in drop tower. The drop interface is very sensitive to the G-Jitter, thus a good microgravity level and a low noise is required to perform accurate contact angle measurements and to observe drop interface without gravity influence. The drop creation phase influence also the final contact angle, experiments will be perform in microgravity to focus on the possible explanation of the contact angle difference observed (gas trapped under the drop, physico-chemical interactions...). Also, a possible explanation for the change in drop contact angle can be found in the drop pressure change through the gravitational pressure.

\section{Nomenclature}

\section{Roman letters}

$\begin{array}{ll}\text { Bo } & \text { Bond number }\left[\frac{g \Delta \varrho D_{0}^{2}}{\sigma}\right] \\ D & \text { Wetting diameter }(\mathrm{m}) \\ g & \text { Gravitational constant: } 9.81 \mathrm{~m} \mathrm{~s}^{-2} \\ h & \text { Drop height }(\mathrm{m}) \\ L_{\mathrm{c}} & \text { Capillary length }(\mathrm{m}) \\ P & \text { Pressure }(\mathrm{Pa}) \\ Q & \text { Injection mass flow rate }\left(\mathrm{kg} \mathrm{s}^{-1}\right) \\ R & \text { Drop radius }(\mathrm{m}) \\ S & \text { curvilinear coordinate }(\mathrm{m}) \\ T & \text { Temperature }\left({ }^{\circ} \mathrm{C}\right) \\ U & \text { Hole exit fluid velocity }\left(\mathrm{m} \mathrm{s}^{-1}\right) \\ x & \text { polar coordinate }(\mathrm{m}) \\ z & \text { Polar coordinate }(\mathrm{m})\end{array}$

\section{Greek letters}

$\theta \quad$ Drop contact angle $\left({ }^{\circ}\right)$

$\phi \quad$ Angle used in the polar coordinate (-)

$\sigma$ Surface tension $\left(\mathrm{N} \mathrm{m}^{-1}\right)$

$\varrho \quad$ Fluid density $\left(\mathrm{kg} \mathrm{m}^{-3}\right)$ 
Acknowledgements We would like to thank the "Centre National d'Etudes Spatiales" for the financial assistance for the campaign PF72 realized at Bordeaux, Merignac, France. Also, we would like to thank Novespace for their assistance during the campaign. This research was partly supported by the National Natural Science Foundation of China (Grant No. 10772185). We would like also to thanks Pascale Aussillous for the fruitful discussions on sessile drops.

\section{References}

Aussillous, P.: Les gouttes enrobees, pp. 30-33. Ph.D. thesis, Universite Paris VI (2002)

Bernardin, J.D., Mudawar, I., Walsh, C.B., Franses, E.I.: Contact angle temperature dependence for water droplets on practical aluminum surfaces. Int. J. Heat Mass Transfer 40(5), 1017-1033 (1997)

Bigioni, T.P., Lin, X.M., Nguyen, T.T., Corwin, E.I., Witten, T.A., Jaeger, H.M.: Kinetically driven self assembly of highly ordered nanoparticle monolayers. Nat. Mater. 5, 265-270 (2006)

Bourges-Monnier, C., Shanahan, M.: Influence of evaporation on contact angle. Langmuir 11, 2820-2829 (1995)

Chandra, S., Di Marzo, M., Qiao, Y.M., Tartarini, P.: Effect of liquid solid contact angle on droplet evaporation. Fire Saf. J. 27, 141-158 (1996)

Frassy, J., Lecot, C., Delattre, C., Soucemarianadin, A.: Droplet evaporation on solid substrates of different wetting behaviour. SHF Microfluidics 2006 (2006)

Gajewski, A.: Contact angle and sessile drop diameter hysteresis on metal surfaces. Int. J. Heat Mass Transfer 51, 4628-4636 (2008)

Grandas, L.: Evaporation d'une goutte sessile: etude experimentale des transferts de chaleur et de masse. Ph.D. thesis, Universite de Provence (2004)
Grandas, L., Reynard, C., Santini, R., Tadrist, L.: Experimental study of the evaporation of a sessile drop on a heat wall. Int. J. Therm. Sci. 44(2), 137-146 (2005)

Kulinich, S.A., Farzaneh, M.: Effect of contact angle hysteresis on water droplet evaporation from super-hydrophobic surfaces. Appl. Surf. Sci. 255, 4056-4060 (2009)

Letellier, P., Mayaffre, A., Turmine, M.: Drop size effect on contact angle explained by nonextensive thermodynamics. Young's equation revisited. J. Colloid Interf. Sci. 314, 604614 (2007)

Ning, Q., Zhu, Z.Q., Li X.T., Yu, Q., Yuan, Z.F.: Determine the surface tension and contact angle of drop by image processing method. Chin. J. Space Sci. 28, 74-79 (2008)

Panwar, A.K., Barthwal, S.K., Ray, S.: Effect of evaporation on the contact angle of a sessile drop on solid substrates. J. Adhes. Sci. Technol. 17(10), 1321-1329 (2003)

Ponter, A.B., Boyes, A.P.: The relation between contact angle and drop size for water at its boiling point for a pressure range 50-760 Torr. Can. J. Chem. 50, 2419 (1972)

Rotenberg, Y., Boruvka, L., Neumann, A.W.: Determination of surface tension and contact angle from the axisymmetric fluid interfaces. J. Colloid Interf. Sci. 93, 169-183 (1983)

Takata, Y., Hidaka, S., Cao, J.M., Nakamura, T., Yamamoto, H., Masuda, M., Ito, T.: Effect of surface wettability on boiling and evaporation. Energy : (Oxford) 30(2-4), 209-220 (2005)

Whyman, G., Bormashenko, E.: Oblate spheroid model for calculation of the shape and contact angles of heavy droplets. J. Colloid Interface Sci. 331, 174-177 (2009)

Yekta-Fard, M., Ponter, A.B.: The influence of vapor environment and temperature on the contact angle drop size relationship. J. Colloid Interf. Sci. 126, 134-140 (1989)

Zhang, N., Chao, D.F.: Flow visualization in evaporating liquid drops and measurement of dynamic contact angles and spreading rate. J. Flow Vis. Image Process. 8(2-3), 303-312 (2001) 